\title{
A Preliminary Study into Emergent Behaviours in a Lattice of Interacting Nonlinear Resonators and Oscillators
}

\author{
Michel F. Randrianandrasana ${ }^{1 \mathrm{a}}$, Xueyong $\mathrm{Wei}^{2 \mathrm{~b}}$, David Lowe ${ }^{3 \mathrm{a}}$ \\ ${ }^{a}$ Nonlinearity and Complexity Research Group, Aston University, Birmingham, UK \\ ${ }^{b}$ School of Mechanical Engineering, University of Birmingham, UK
}

\begin{abstract}
Future sensor arrays will be composed of interacting nonlinear components with complex behaviours with no known analytic solutions. This paper provides a preliminary insight into the expected behaviour through numerical and analytical analysis. Specifically, the complex behaviour of a periodically driven nonlinear Duffing resonator coupled elastically to a van der Pol oscillator is investigated as a building block in a 2D lattice of such units with local connectivity. An analytic treatment of the 2-device unit is provided through a two-time-scales approach and the stability of the complex dynamic motion is analysed. The pattern formation characteristics of a $2 \mathrm{D}$ lattice composed of these units coupled together through nearest neighbour interactions is analysed numerically for parameters appropriate to a physical realisation through MEMS devices. The emergent patterns of global and cluster synchronisation are investigated with respect to system parameters and lattice size.
\end{abstract}

Keywords: Duffing resonator; van der Pol oscillator; MEMS; emergent collective behaviour

\section{Introduction}

Real biosensor networks, such as the mammalian olfactory bulb as an exemplar system, and neurally-integrated autonomous communication networks in many nervous systems, share common characteristics and behaviours which are still not understood. Such systems

\footnotetext{
${ }^{1}$ Corresponding author. Email: randrimf@aston.ac.uk

${ }^{2}$ Email: x.wei.2@bham.ac.uk

${ }^{3}$ Email: d. lowe@aston.ac.uk
} 
are composed of interconnected relatively simple dynamical systems, but which are nonlinear, or interact nonlinearly and permit dynamical interactions on multiple time scales. Such systems exhibit a huge diversity of behaviours and produce emergent and macroscopic properties which are responsive in ways they interact with the environment that are unfamiliar to traditional engineering sensor design.

For example [1], biophysical models to explain the gamma band oscillation in the olfactory bulb based on coupled subthreshold oscillators have been shown to exhibit synchronisation and coherence effects when driven with correlated inputs. Similarly, in recent models of temporal coding [2], competition for coherence driven by increased phase velocity of neural units in cases of coherent input from the connected neurons leads to different perspectives on assembly formation in collections of coupled oscillator neurons. This assembly formation suggests novel mechanisms for pattern recognition. The ability of a network of coupled nonlinear oscillators to develop emergent behaviour including topological organisation, associative memory and gamma band synchronisation, has already been used to illustrate a computational capability for abstract pattern recognition [3].

However, a major query is whether we can learn from such biological sensor and communication networks to design more robust biomorphically engineered sensor, communication and computing arrays in recent technologies such as micro and nano-mechanical silicon-based systems.

Motivated by the examples presented above, we are investigating the effects of Si-based sensor arrays of coupled microelectromechanical oscillators and resonators. We are interested in the characterisation and detection of novel self-organising response patterns as prototype simultaneous sensing and computing primitives in a pattern recognition system. Whereas traditional design rules would attempt to create an array of isolated and linear-response sensors, the biomorphic design implies interaction and nonlinear oscillators. This allows the emergence of a much richer range of behaviours of the array beyond that expected in a traditional design strategy, including macroscopic and cluster synchronisation, dynamic pattern flows, extinction, and adaptation. These different modes of operation could in the future be used for adaptive integrated sensing and computing. 
As a preliminary stage to investigating whether MEMS arrays are capable of exhibiting and thus exploiting similar ranges of behaviour to the biological counterparts, this paper focusses on the range of emergent properties of coupled oscillator arrays as expected in real microelectromechanical arrays where the simple 'neuron' oscillator elements are allowed to interact locally with neighbouring elements. Each neuron element is a simple nonlinear dynamical system characterised by self-sustaining and damped oscillator.

As already noted, in biological systems of coupled oscillatory devices, the interaction permits macroscopic temporal characteristics such as common locking of the phase or amplitude into synchronised states. In order to understand and predict the emergent phenomena of these systems, different mathematical models of chains and arrays of coupled resonators or oscillators have been constructed and studied [4]. In a strongly coupled system, the synchronisation effects may be observed globally in all elements $[5,6,7,8,9,10,11,12]$ or locally in clusters of neighbouring elements $[13,14,15,16,17,18]$ (cluster synchronisation).

To extend these recent activites and also to link to recent mathematical work on coupled oscillator array systems, in this paper, the collective behaviour of alternating Duffing resonators and van der Pol oscillators elastically coupled in a square lattice is numerically studied with typical MEMS parameters. The fundamental study of coupled nonlinear oscillators is important in understanding the emergent behaviour of complex dynamical systems and developing novel M/NEMS devices [19, 20, 21]. The analysis of a simpler case as the building block can help to gain insight into larger complicated systems [22, 23]. Among the building blocks studied in the literature, the essential elements are either self-sustained oscillators (van der Pol oscillators) or dissipative oscillators (Duffing-type resonators) and the most intensively studied cases are the coupled van der Pol and the coupled Duffing oscillators $[24,25,26]$. To the best of our knowledge, less has been done in a dynamic system consisting of a Duffing-type resonator coupled to a self-excited oscillator. For instance, the dynamics of such a system was numerically studied using three control parameters, namely two damping coefficients and a coupling constant in [27] where three different synchronisation phenomena were found and a chaotic state was clearly identified in the phase diagram. The dynamics of such an undriven system was also studied in the form of a van der Pol 
oscillator with a nonlinear restoring force coupled to a Duffing resonator through a mixed velocity and displacement coupling condition [28] and an inertial force condition [29]. Analytic solutions of stable oscillation states were derived in both cases and a chaotic behaviour

was also observed. This paper is an extension of the results in [30] concerning a system of driven Duffing resonators coupled to van der Pol oscillators. More complex dynamics could appear in such an externally driven nonlinear system of two elastically coupled oscillators of different types of attractor.

Therefore, the dynamics of such a system externally driven by a periodic force will considered first in section 2 followed by a description of the architecture of the square lattice of resonators and oscillators in section 3. Some characterisations of synchronisation will also be described and the effect of parameter variation on the behaviour of the system will be discussed along with different configurations and sizes of the lattice in the latter section.

\section{Single unit of a Duffing resonator coupled to a van der Pol oscillator}

In this section, the behaviour of a periodically driven Duffing resonator coupled to a van der Pol oscillator is discussed. A good understanding of the behaviour of those two coupled elements is necessary as they consitute the building block of a more complicated system such as a square lattice of Duffing resonators coupled to van der Pol oscillators which will be investigated in the next section. An analytical treatment of the two coupled elements is possible due to the small size of the system. This can be done through the multiple time scales analysis [31] which is derived from perturbation analysis. The linear stability of the system can also be analysed through the eigenvalues of the Jacobian matrix of the system. The study of the presence of chaos in the system can be conducted through bifurcation analysis or through the computation of its maximum Lyapunov exponent. These different aspects of the analysis will be discussed in the following.

The motion of the coupled dynamical system in dimensionless form is described by the 
following set of equations:

$$
\begin{aligned}
\ddot{x_{1}}+\gamma_{1} \dot{x_{1}}+x_{1}+\delta x_{1}^{3} & =\kappa\left(x_{2}-x_{1}\right)+f_{d} \cos \left(\frac{\omega_{d}}{\omega_{1}} \tau\right), \\
\ddot{x_{2}}+\gamma_{2}\left(x_{2}^{2}-1\right) \dot{x_{2}}+\left(\frac{\omega_{2}}{\omega_{1}}\right)^{2} x_{2} & =\kappa\left(x_{1}-x_{2}\right)
\end{aligned}
$$

where, $x_{1}, \gamma_{1}, \omega_{1}$ and $x_{2}, \gamma_{2}, \omega_{2}$ are the displacement, damping coefficient and fundamental frequency of the Duffing resonator and the van der Pol oscillator respectively. $f_{d}$ and $\omega_{d}$ are the amplitude and frequency of the external driving force. $\delta$ is the nonlinearity of the Duffing resonator and $\kappa$ is the coupling stiffness between the two coupled elements. When $\kappa$ diminishes to zero, Eqs.(1) uncouple to yield a driven Duffing resonator and a van der Pol oscillator whose limit cycle is determined by $\gamma_{2}$. For the case of $f_{d}=0$, Eqs.(1) can be considered as mutually coupled Duffing resonator driven by a van der Pol oscillator.

Usually, MEMS resonators and oscillators are working in a high Q scenario while they are driven into their resonances with a small force. However, if the Duffing resonator and van der Pol oscillator are working at different fundamental frequencies (the nonresonant case $\left.\omega_{1} \neq \omega_{2}\right)$, it can be proved that the dynamic systems are uncoupled and the time evolution of the amplitudes is the same as the classical driven Duffing resonator and van der Pol oscillator. In the following section, we consider only the case where both the internal and external resonances coincide $\left(\omega_{1}=\omega_{2}\right)$. As the coupling stiffness varies across a large range, we deal separately with the system of weak coupling $(\kappa \ll 1)$ and strong coupling $(\kappa \gg 1)$.

When the van der Pol oscillator is weakly connected to the Duffing resonator, we rewrite Eqs.(1) as follows:

$$
\begin{aligned}
\ddot{x_{1}}+\epsilon c_{1} \dot{x_{1}}+x_{1}+\epsilon \alpha x_{1}^{3} & =\epsilon \beta\left(x_{2}-x_{1}\right)+\epsilon F \cos (\Omega \tau), \\
\ddot{x_{2}}+\epsilon c_{2}\left(x_{2}^{2}-1\right) \dot{x_{2}}+x_{2} & =\epsilon \beta\left(x_{1}-x_{2}\right)
\end{aligned}
$$

where $\gamma_{1}=\epsilon c_{1}, \gamma_{2}=\epsilon c_{2}, \delta=\epsilon \alpha, \kappa=\epsilon \beta, f_{d}=\epsilon F, \Omega=\frac{\omega_{d}}{\omega_{1}}$ and $\epsilon$ is a small parameter.

The response of the system driven near the primary resonance for small dissipation and driving can be calculated from Eqs.(2) using the standard two time scales method 
[31]. Briefly, we introduce the slowly varying complex mode amplitudes $A_{1}, A_{2}$ using $x_{1}=$ $\Re\left(A_{1} e^{i \tau}\right)$ and $x_{2}=\Re\left(A_{2} e^{i \tau}\right)$, substitute into the Eqs.(2), and retain the only near resonant terms. This reduce the Eqs.(2) to

$$
\begin{aligned}
2 A_{1}^{\prime}+c_{1} A_{1}-3 i \alpha\left|A_{1}\right|^{2} A_{1}+i \beta\left(A_{2}-A_{1}\right)-\frac{i}{2} F e^{i \sigma \tau_{1}} & =0 \\
2 A_{2}^{\prime}+c_{2}\left(\left|A_{2}\right|^{2}-1\right) A_{2}+i \beta\left(A_{1}-A_{2}\right) & =0 .
\end{aligned}
$$

We have introduced the frequency detuning $\sigma$ to characterize the closeness of driving frequency to the fundamental frequency, given by $\Omega=1+\epsilon \sigma$ and the derivative are with respect to the slow time scale $\tau_{1}$.

Expressing the slowly varying complex mode amplitudes $A_{1}$ and $A_{2}$ in polar form:

$$
\begin{aligned}
& A_{1}=a_{1} e^{i \theta_{1}}, \\
& A_{2}=a_{2} e^{i \theta_{2}},
\end{aligned}
$$

The amplitude response of the steady-state motions of Eqs. 3 are given by the following set of algebraic equations,

$$
\begin{aligned}
\left(a_{1}(\beta-2 \sigma)+3 a_{1}^{3} \alpha-\frac{a_{2}^{2}(\beta-2 \sigma)}{a_{1}}\right)^{2}+\left(a_{1} c_{1}-\frac{a_{2}^{2} c_{2}}{a_{1}}\left(1-a_{2}^{2}\right)\right)^{2} & =\frac{1}{4} F^{2} . \\
(\beta-2 \sigma)^{2} a_{2}^{2}+c_{2}^{2} a_{2}^{2}\left(1-a_{2}^{2}\right)^{2} & =a_{1}^{2} \beta^{2} .
\end{aligned}
$$

The corresponding frequency-response curves, for the variation of the amplitude $A$ with the frequency $\Omega$, of the Duffing resonator and van der Pol oscillator are shown in Figure 1, where the coupling strength $\kappa=0.002$. Equation (5b) shows Duffing-type behaviour of the van der Pol oscillator, provided that the coupling and damping coefficients are not zero. If the coupling effect diminishes, then equation (5a) will be reduced to the frequency-amplitude modulation of a normal Duffing resonator.

If the coupling stiffness $\kappa$ is not the same order of magnitude as the other parameters, 


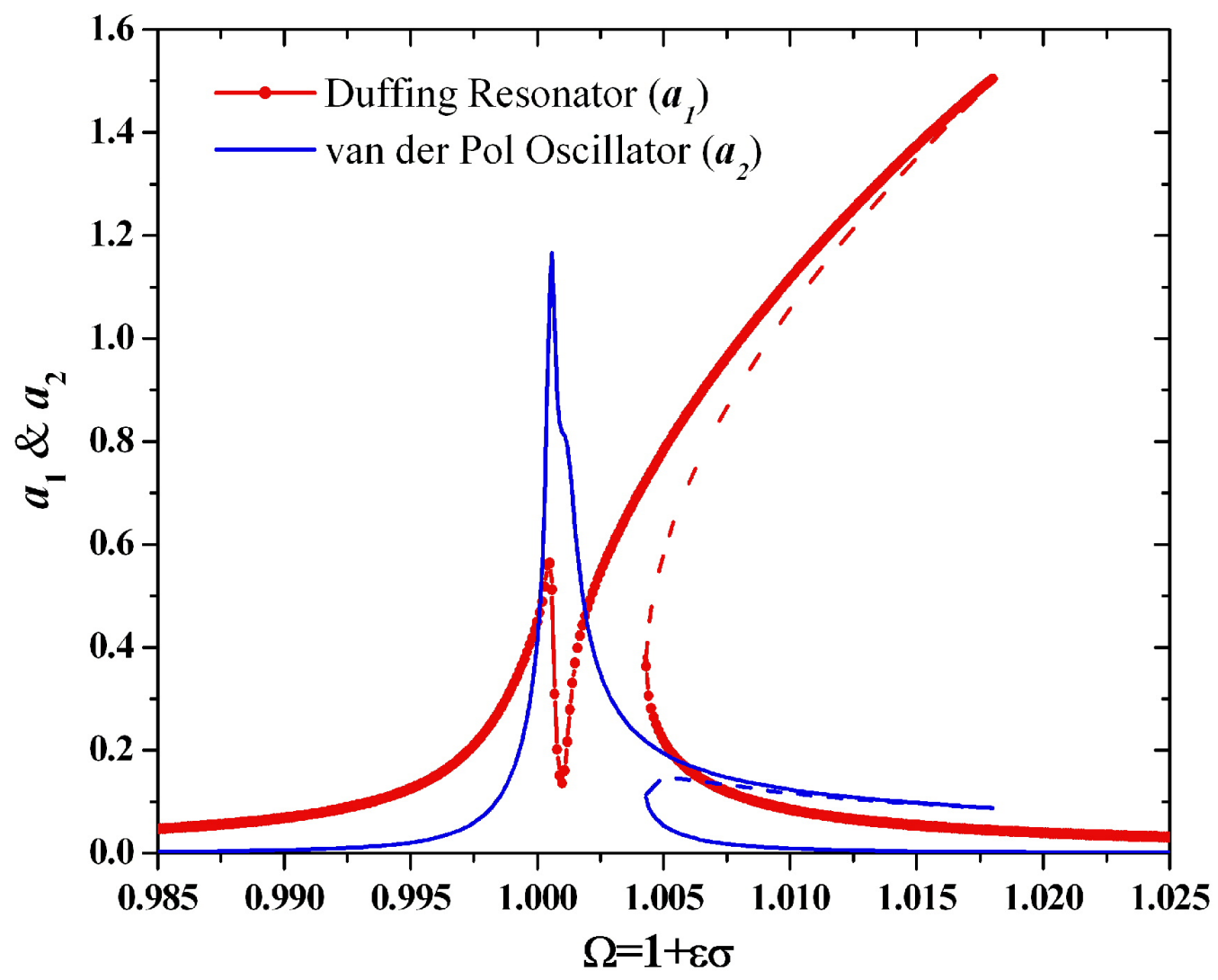

Figure 1: Frequency-response curves of the driven Duffing resonator (red curve) weakly coupled to a van der Pol oscillator (blue curve). The unstable branches are drawn in dashed lines. The system parameters are $\gamma_{1}=\gamma_{2}=0.001, \delta=0.005, \kappa=0.002, f_{d}=0.003$. 
the above analysis does not hold, so a modified analysis considering strong coupling is given as follows. When $\kappa$ is larger than 4.5, for example, $\frac{1}{2 \kappa+1}$ is less than 0.1. Therefore, We choose $\epsilon=\frac{1}{2 \kappa+1}$ and assume all other coefficients are small as defined above.

Then Eqs.(1) can be rewritten as,

$$
\begin{aligned}
\ddot{x}_{1}+\ddot{x_{2}}+\epsilon c_{1} \dot{x_{1}}+\epsilon c_{2}\left(x_{2}^{2}-1\right) \dot{x_{2}}+x_{1}+x_{2}+\epsilon \alpha x_{1}^{3}=\epsilon F \cos (\Omega \tau), \\
\epsilon\left(\ddot{x}_{1}-\ddot{x_{2}}\right)+\epsilon^{2} c_{1} \dot{x_{1}}-\epsilon^{2} c_{2}\left(x_{2}^{2}-1\right) \dot{x_{2}}+x_{1}-x_{2}+\epsilon^{2} \alpha x_{1}^{3}=\epsilon^{2} F \cos (\Omega \tau) .
\end{aligned}
$$

Applying the standard two-scales analysis, we found that the Duffing resonator and the van der Pol oscillator are still completely synchronised in the first order approximation when they are strongly coupled. The Eqs.6 reduces to the following equation by imposing secular conditions:

$$
4 A^{\prime}+\left(c_{1}-c_{2}\right) A+c_{2} A|A|^{2}-3 i \alpha A|A|^{2}+i \frac{1}{2} F e^{i \sigma \tau_{1}}=0
$$

For the steady state solutions, we obtain the following algebraic equation describing the amplitude response of the system:

$$
a^{2}\left(-4 \sigma+3 \alpha a^{2}\right)^{2}+a^{2}\left(c_{1}-c_{2}+c_{2} a^{2}\right)^{2}=\frac{1}{4} F^{2}
$$

The corresponding frequency-response curve of the system is shown in Figure 2, where the dashed lines correspond to the unstable branches and the solid lines are the stable branches. The shark's thin shape of the curve is typical of a single driven Duffing resonator.

And the general solution of the strongly coupled Duffing resonator and van der Pol oscillator in the first order approximation is given by

$$
x_{1}=x_{2}=\left(a e^{i(\Omega \tau+\gamma)}+\frac{1}{16} \epsilon\left(\alpha-c_{2} i\right) a^{3} e^{3 i(\Omega \tau+\gamma)}+c c\right)
$$

which shows that in the case of strong coupling, the van der Pol oscillator is completely synchronized to the Duffing resonator and their oscillations are also locked to the external driving force by a phase lag. This is confirmed numerically, for a coupling strength $\kappa=8$, by the phase portraits of Figure 3 where the trajectories of the Duffing resonator and van der 


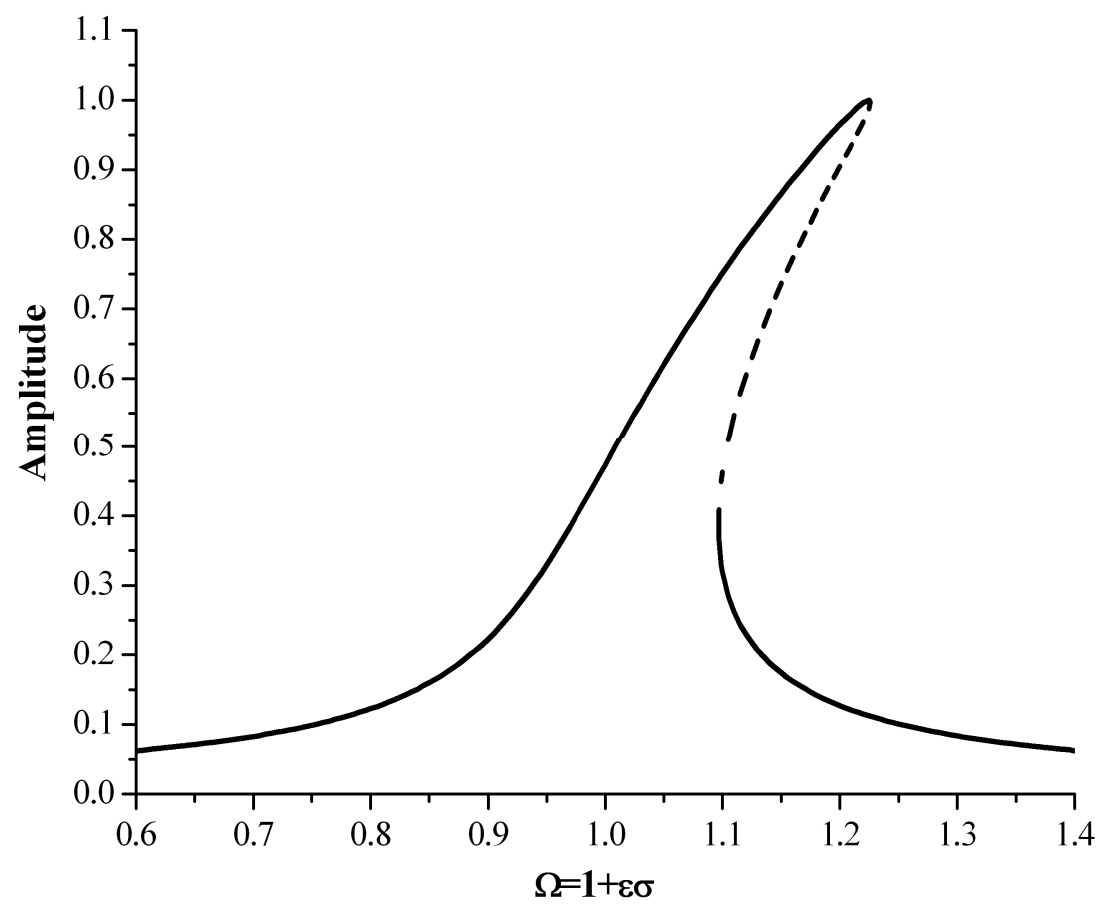

Figure 2: Frequency-response curve of the driven Duffing resonator strongly coupled to a van der Pol oscillator. The system parameters are $\gamma_{1}=0.1, \gamma_{2}=0.05, \delta=0.3, \kappa=8, f_{d}=0.2$. 
Pol oscillator are plotted in phase space. Figure 3(a) and 3(b) show the early complicated behaviour, also called initial transient, of the Duffing resonator and van der Pol osccillator during the first hundred periods of oscillations. After an initial transient of 2000 periods, the Duffing resonator and the van der Pol oscillator become complete synchronised as shown in Figure 3(c).

The frequency-response curves obtained previously showed the steady state approximate solutions of the coupled systems given by the multiple time scales analysis. For the weak coupling case, the system can be driven into multiple states as shown in Figure 1 where the stable (solid lines) and unstable (dashed lines) branches of the coupled system. The stability is obtained through the analysis of the Jacobian matrix. As can be expected, the lower branches, in red colour, of the hysteresis for the Duffing resonator and van der Pol oscillator respectively are unstable for the system parameters that have been chosen. One can then expect that in a lattice of such a unit, more complicated behaviour can appear and it is therefore interesting to investigate their collective behaviour. The system also displays interesting phenomena such as an anti-resonance at $\Omega \approx 1$ and a shoulder in the curve which is due to a stagnant reponse of the van der Pol oscillator. For the strong coupling case, the system behaves like a driven Duffing resonator as shown in Figure 2 where the middle branch is unstable. A further study of the dependence of the system dynamics on its parameters was conducted using a bifurcation analysis [32]. Now that some insights about the behaviour of the two coupled elements have been gained, a new topology in which the Duffing resonators and van der Pol oscillators are coupled alternatively in a square lattice will be considered in the next section.

\section{Collective behaviour in a square lattice of driven Duffing resonators coupled to van der Pol oscillators}

The global and local synchronisation of a square lattice composed of alternating Duffing resonators and van der Pol oscillators coupled through displacement is studied in this section. The lattice acts as a sensing device in which the input signal is characterised by an external driving force that is injected into the system through a subset of the Duffing resonators. 


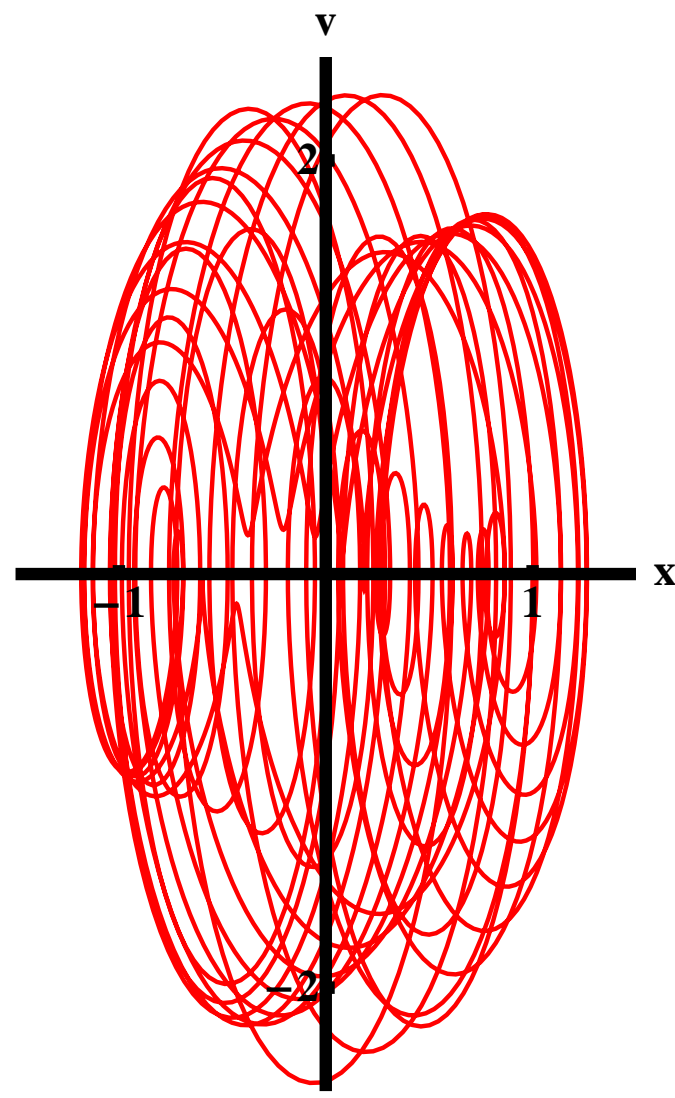

(a) Duffing (Duff)

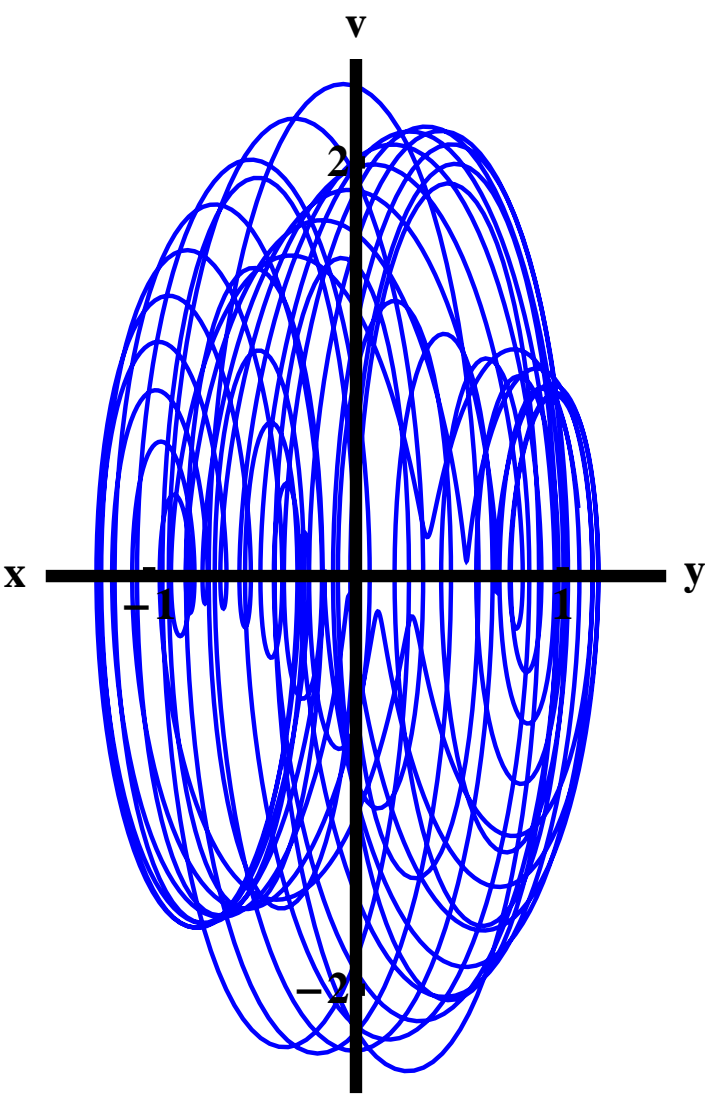

(b) van der Pol (VdP)

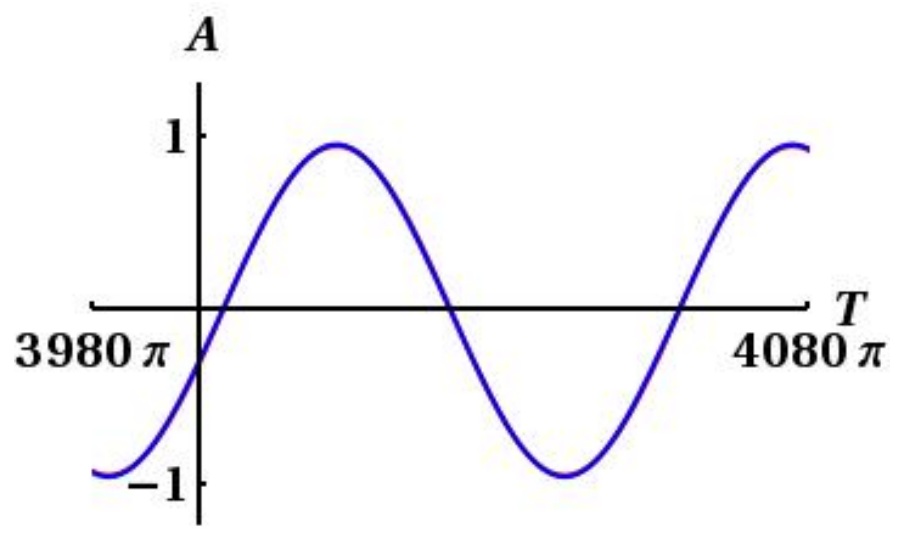

(c) Duff + VdP

Figure 3: Phase portraits and time series of a driven Duffing resonator (red) strongly coupled to a van der Pol oscillator (blue). The system parameters are $\gamma_{1}=0.1, \gamma_{2}=0.05, \delta=0.3, \kappa=8, f_{d}=0.2$. Both elements show an early complicated behaviour, also called initial transient, during the first hundred periods of oscillations (3(a) and 3(b) ). After an initial transient of 2000 periods, the Duffing resonator and the van der Pol oscillator become synchronised as shown by the time series in 3(c). 
The parameters of the system are taken from MEMS devices. The effects of the system parameters, the lattice architecture and size are discussed.

In studies of the collective behaviour of oscillatory systems, when some elements share similar temporal characteristics such as the phase or amplitude, they are generally said to be synchronised. The synchronisation effects may be observed globally in all elements $[5,6,7,8,9,10,11,12]$ or locally in clusters of neighbouring elements $[13,14,15,16,17,18]$. In this section, the collective behaviour of alternating Duffing resonators and van der Pol oscillators coupled through displacement in a square lattice is studied. The architecture of the lattice of resonators and oscillators along with some characterisations of synchronisation will be described first. Then the effect of some parameters on the behaviour of the system will be discussed followed by different configurations and sizes of the lattice.

\subsection{Experimental setup}

The architecture of the network of oscillators consists of a square lattice of alternating Duffing resonators and van der Pol oscillators in a checkerboard configuration as shown in Figure 4. The dimensionless equations governing the coupled systems are:

$$
\begin{aligned}
\ddot{x}+\gamma \dot{x}+x+\delta x^{3} & =\kappa\left(x_{i, j+1}+x_{i, j-1}+x_{i-1, j}+x_{i+1, j}-4 x_{i, j}\right), \\
\ddot{x}+\gamma\left(x^{2}-1\right) \dot{x}+x & =\kappa\left(x_{i, j+1}+x_{i, j-1}+x_{i-1, j}+x_{i+1, j}-4 x_{i, j}\right),
\end{aligned}
$$

where $\gamma$ is the damping of the Duffing resonator and the van der Pol oscillator, $\delta$ is the cubic nonlinear spring constant of the Duffing resonator and $\kappa$ is the coupling strength between the resonators and oscillators. The element $x_{i, j}$ represents the displacement of the resonator or oscillator located at the $i$-th row and $j$-th column in the square lattice.

Since the array of oscillators will act as a sensing device, it is usually subject to some external signal that can be represented as a driving force or energy for the array. The equations for the driven Duffing resonator and van der Pol oscillator respectively are therefore given below:

$$
\ddot{x}+\gamma \dot{x}+x+\delta x^{3}=F_{d} \cos (\Omega \tau)
$$


and

$$
\ddot{x}+\gamma\left(x^{2}-1\right) \dot{x}+x=F_{d} \cos (\Omega \tau),
$$

where $F_{d}$ is the normalised driving force.

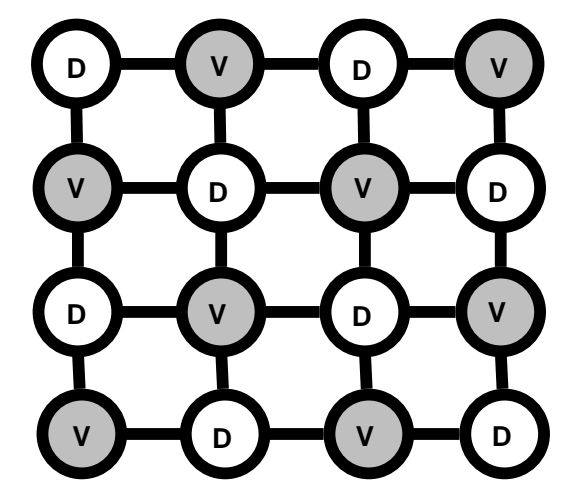

Figure 4: Square lattice of alternating Duffing resonators and van der Pol oscillators.

In this section, the synchronous behaviour of the resonators and oscillators in the square lattice will be characterised in terms of their global and local synchronisations. In the first case, the resonators and oscillators will mainly be considered as one group whereas the elements in the array can be gathered in different subgroups in the second case. The global coherence of the array will be measured by a synchronisation index based on the normalised entropy [33]:

$$
\tilde{\rho}=\frac{S_{\max }-S}{S_{\max }}
$$

where $S=-\sum_{k=1}^{N} \rho_{k} \ln \rho_{k}$ is the entropy of the distribution of the instantaneous phase or the amplitude of the oscillators, which is given by their respective histograms from which the probabilities $\rho_{k}$ are computed, and $S_{\max }=\ln N$, where $N$ is the number of bins of the histogram. $0 \leq \tilde{\rho} \leq 1$ and $\tilde{\rho}=0$ means that the array is not coherent or in total disorder, whereas $\tilde{\rho}=1$ indicates that all the oscillators in the array are perfectly synchronised. Besides full synchronisation it is also interesting to consider another aspect of synchronisation namely the cluster synchronisation where the oscillators involved in the same cluster have identical temporal dynamics and completely synchronise between each other. Unlike the global synchronisation case, no cluster synchronisation index is available yet. Therefore, 
only a visual inspection of the displacement and phases of the elements in the lattice will be carried out. To this end, phase images and polar plots of the elements of the square lattice will be produced. The phase and polar plots will be complementary as the phase images will help to locate the resonators and oscillators within the array while the polar plots will display both the amplitudes and phases of the elements. In the following, the color scale in the phase images lies between $-\pi$ and $\pi$ and the polar plots are normalised in amplitude which means that the maximum amplitude corresponds to a distance of 1 in the unit circle.

\subsection{Parameter effects}

In this section we investigate the effect of different parameters which have an impact on the behaviour of the lattice of oscillators and resonators. These include the normalised coupling strength $\kappa$ between the oscillators, the amplitude $F_{d}$ of the driving force that is injected from one side of the lattice and finally the quality factor $Q$ of each element of the lattice. It should be noted that the quality factor $Q$ of the oscillators and resonators is directly related to their damping $\gamma$ through the expression $\gamma=1 / Q$. It is also important to stress the fact that only the Duffing resonators are driven since they absorb energy whereas the van der Pol oscillators are self-sustained.

In the following, the parameters are given in their dimensionless values. In the context of MEMS devices, the coupling strength $\kappa$ ranges from 0.001 to 1000 . The amplitude $F_{d}$ of the driving force is assumed to be in the range 0.3 to 30. The quality factor $Q$ lies within the interval $[10,10000]$, where the lowest and biggest $Q$ values correspond to the devices being in air and in vacuum respectively.

The coupling strength between the elements of the array has a counter-intuitive effect on the global coherence of the amplitudes of the resonators and oscillators. The lower the coupling the more coherent is the system as shown in Figure 5. This is due to the fact that the value of the normalised entropy is maximum only when all the elements have the same value, or in other words, when there is only one cluster. However, when the coupling strength is high the Duffing resonators and the van der Pol oscillators evolve into two different clusters as shown in Figure 6. This implies that the higher the coupling strength the more synchronised are the Duffing resonators between themselves and so do the van der 


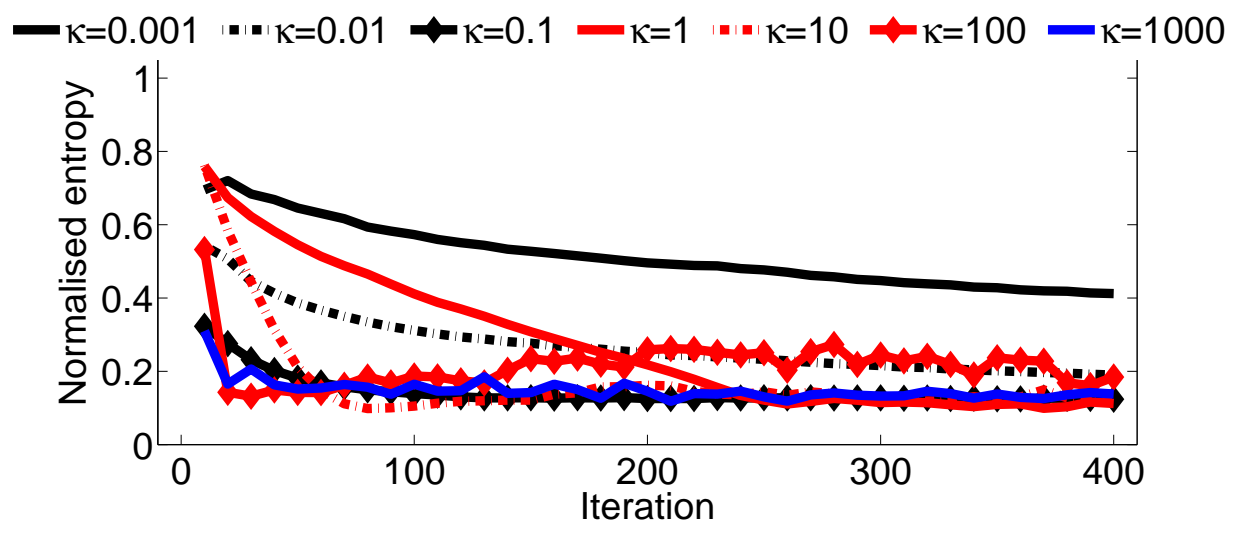

(a) Phase

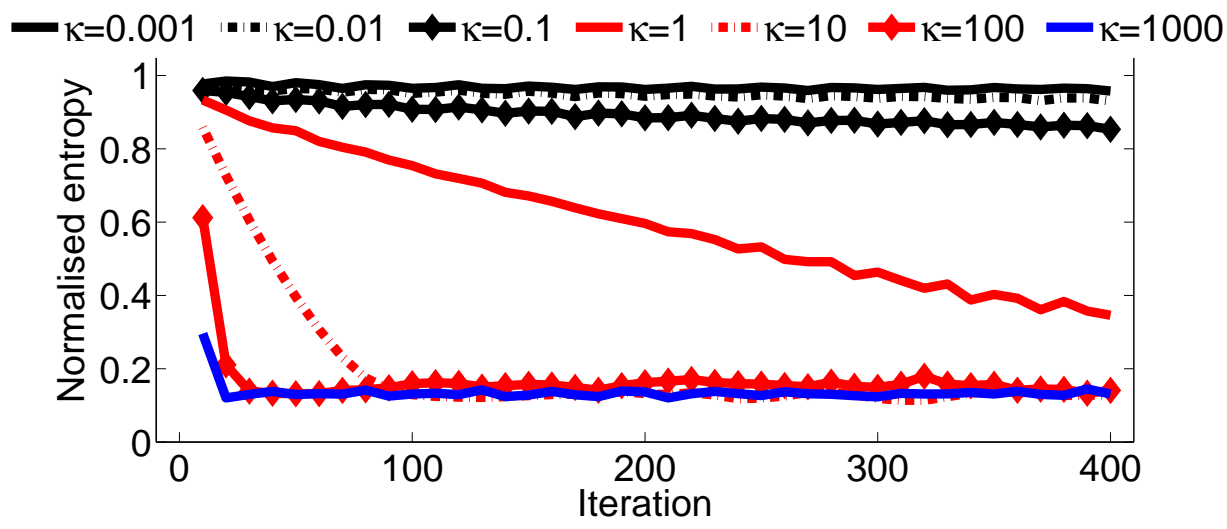

(b) Amplitude

Figure 5: Coupling strength effect on the global coherence of the square lattice. The phase coherence does not seem to have a direct relation with the coupling strength although for a very high coupling $(\kappa=1000)$ the phases of the elements of the lattice tend to converge faster. On the other hand, the amplitude coherence clearly depends on the coupling strength value: the higher the coupling strength, the quicker the convergence into a stable state. It should be noted that the normalised entropy is low when the coupling strength is high because the Duffing resonators and the van der Pol oscillators evolve into two different clusters as shown in Figure 6. The normalised entropy is maximum only when all the elements have the same value or in other words when there is only one cluster. 


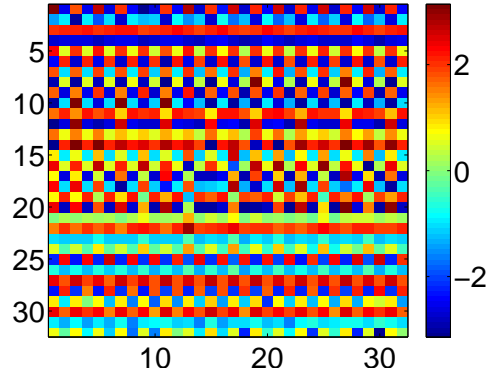

(a) $\kappa=1$

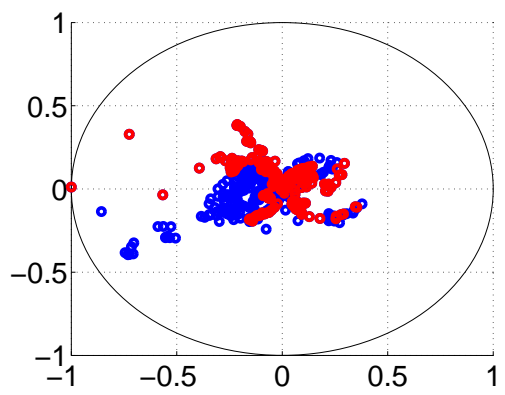

(c) $\kappa=1$

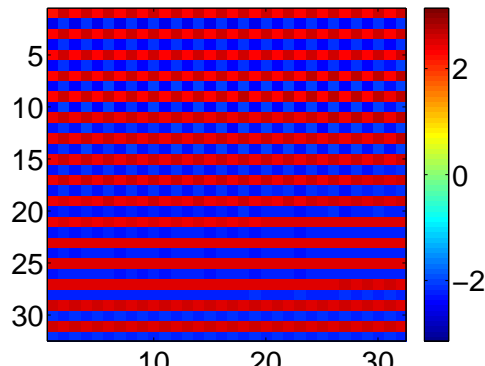

(b) $\kappa=100$

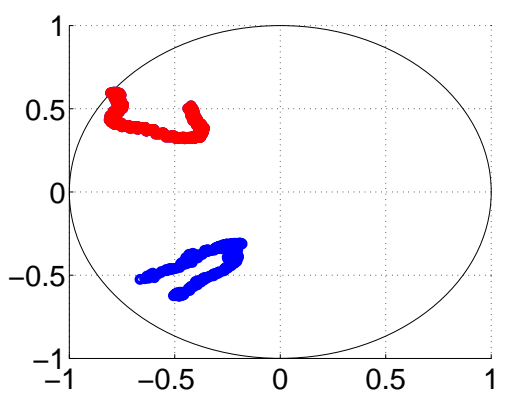

(d) $\kappa=100$

Figure 6: Phase and polar plots of a square lattice of 32 by 32 x-coupled alternating Duffing resonators and van der Pol oscillators driven from the top edge with an energy whose amplitude is $F_{d}=0.3$. The figures are plotted at $t=3600$ with different coupling strength values. At low coupling $(\kappa=1)$ both the phases and amplitudes of the resonators and oscillators seem to be random. For high coupling $(\kappa=100)$ the resonators and oscillators on odd rows are synchronised between them and so are the resonators and oscillators located on even rows. This is an example of distributed cluster synchronisation where there is a division into quasi-independent sublattices. 


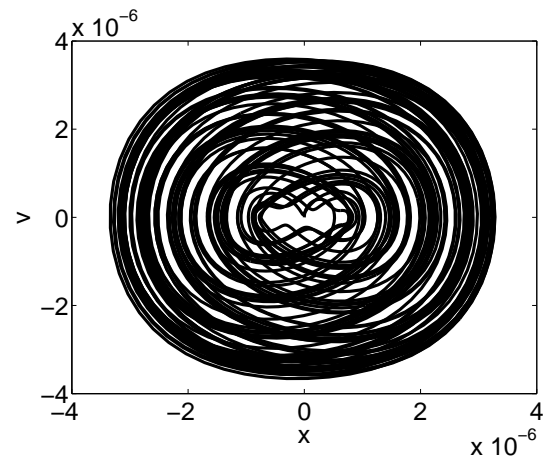

(a) $\operatorname{vdp}(1,1)$

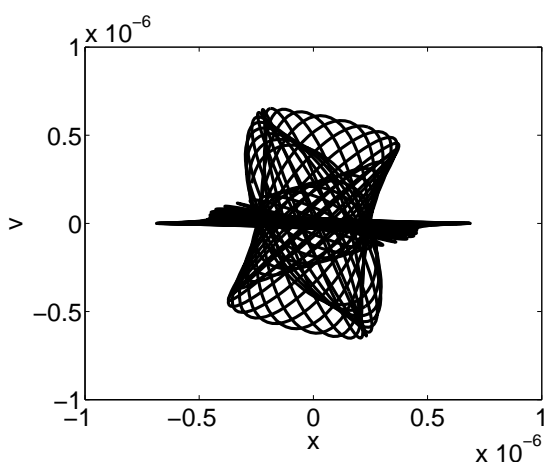

(c) $\operatorname{vdp}(1,17)$

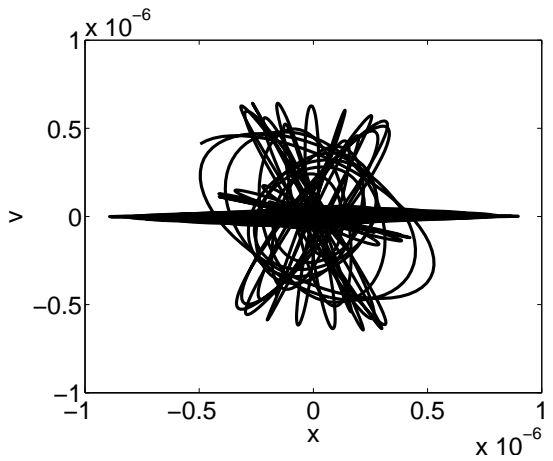

(e) $\operatorname{vdp}(31,17)$

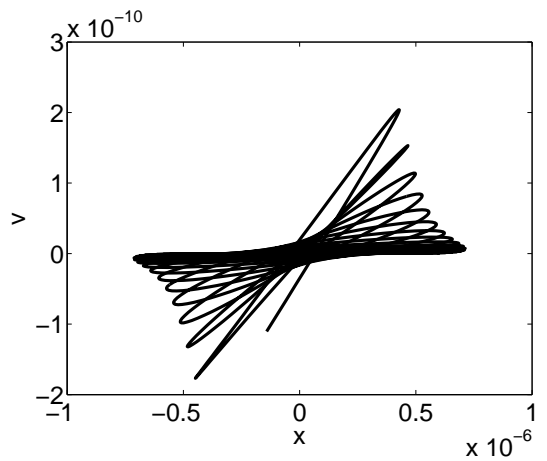

(b) $\operatorname{duff}(1,32)$

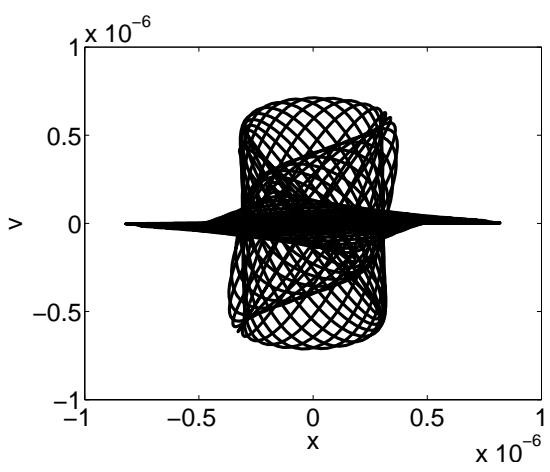

(d) $\operatorname{duff}(1,16)$

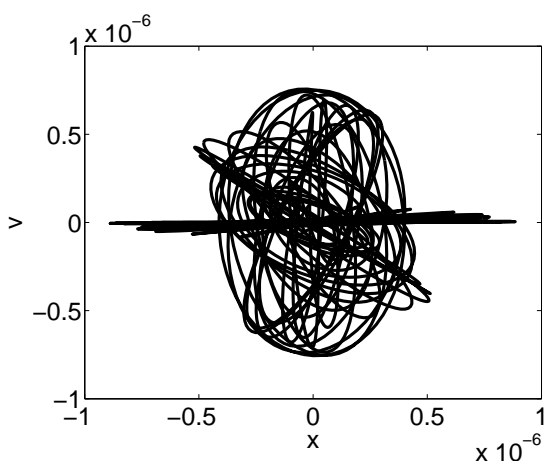

(f) $\operatorname{duff}(31,16)$

Figure 7: Phase portrait in an array of $32 \times 32 \mathrm{x}$-coupled alternating Duffing resonators and van der Pol oscillators. The notation $\operatorname{vdp}(x, y)$ and $\operatorname{duff}(x, y)$ indicates the selected locations $(x, y)$ of a van der Pol or Duffing device. An energy, whose amplitude is $F_{d}=0.3$, is injected from the top edge of the lattice. The coupling strength between the oscillators is $\kappa=1$. The figures show that small coupling induces different types of complicated behaviours. 
Pol oscillators, an observation which cannot be captured by the value of the normalised entropy in this case. The value of the coupling strength has no clear relation with the global phase coherence of the elements although the global phase coherence of the system tends to converge quicker with higher coupling values. More detailed information regarding the cluster synchronisation of the elements in the lattice are given by the phase and polar plots in Figure 6. It can clearly be seen that with low coupling $(\kappa=1)$ the behaviour of the elements is more like random. However, with high coupling $(\kappa=100)$ the phases of the resonators and oscillators located on odd rows, and respectively on even rows, are mutually synchronised. These two distinct clusters are shown in both the phase and amplitude spaces by the polar plot of Figure 6(d).

Regarding the dynamical properties of each single element, it can be noticed from the phase portrait plots in Figure 7, where the displacment $x$ is plotted against the velocity $v$, that different types of complicated behaviours of the elements appear with small coupling $(\kappa=1)$. As the coupling strength increases, more homogeneous behaviours between each type of element are observed.

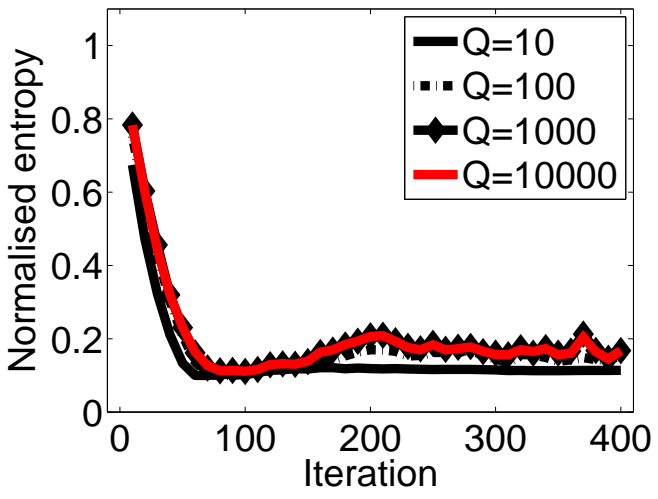

(a) Phase

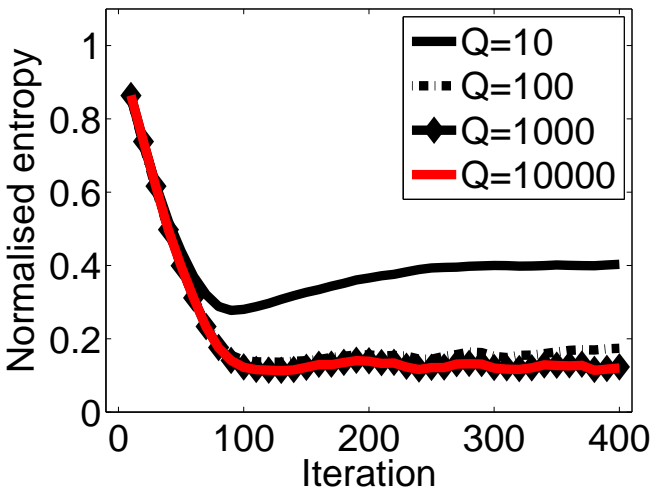

(b) Amplitude

Figure 8: Relation between the quality factor $Q$ of the elements of the square lattice and its global coherence. It can be noticed that the lower the value of $Q$, the worse the phase coherence. A possible explanation of this observation is that for low $Q$ values, the separation between the behaviour of the Duffing resonators and van der Pol oscillators is clearly seen as depicted by Figure 9(c). In contrast, the amplitude coherence of the lattice is improved as the value of $Q$ is decreased.

The global phase coherence of the lattice is not sensitive to the value of the quality factor. 


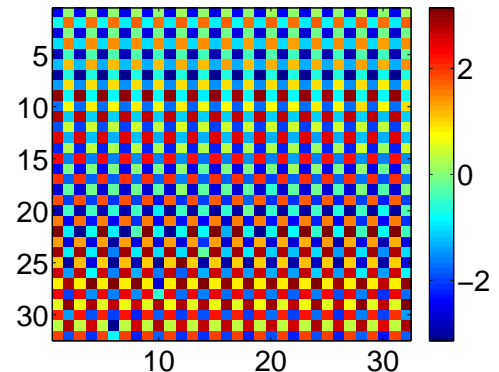

(a) $Q=10$

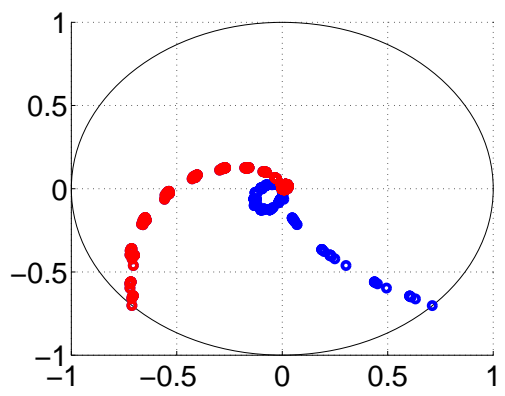

(c) $Q=10$

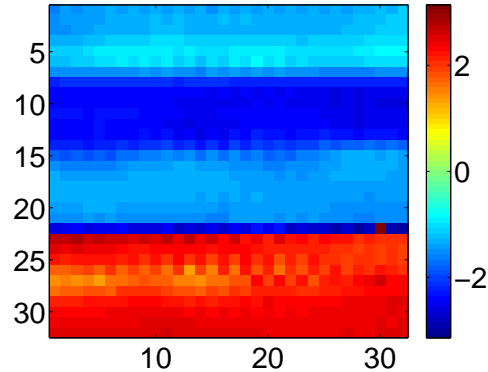

(b) $Q=1000$

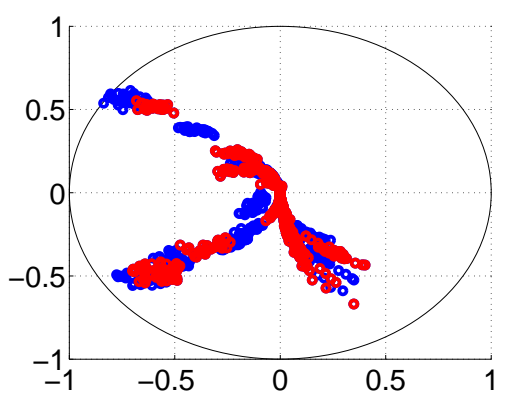

(d) $Q=1000$

Figure 9: Phase and polar plots of a square lattice of 32 by $32 \mathrm{x}$-coupled alternating Duffing resonators and van der Pol oscillators driven from the top edge with an energy whose amplitude is $F_{d}=0.3$. The coupling between the elements is $\kappa=10$ and the figures are plotted at $t=3600$ with different values of the quality factor $Q$. The figures show that when $Q$ is small $(Q=10)$ the Duffing resonators (red circles) and the van der Pol oscilators (blue circles) have two distinct behaviours. However at a high quality factor $(Q=1000)$ they tend to synchronise between them. 
However, for low quality factor value $(Q=10)$ the global amplitude coherence of the system displays better synchronisation behaviour than higher values of $Q$ as shown by Figure 8 . In contrast to the previous full synchronisation results, more distinguishable amplitude and phase clusters appear in the lattice when the quality factor value is high $(Q=1000)$ as can be seen in Figure 9. It should be noted however that for small $Q$ values, the Duffing resonators and van der Pol oscillators have two distinct behaviours.

The system is not sensitive to initial conditions (plots not shown).

\subsection{Size effects}

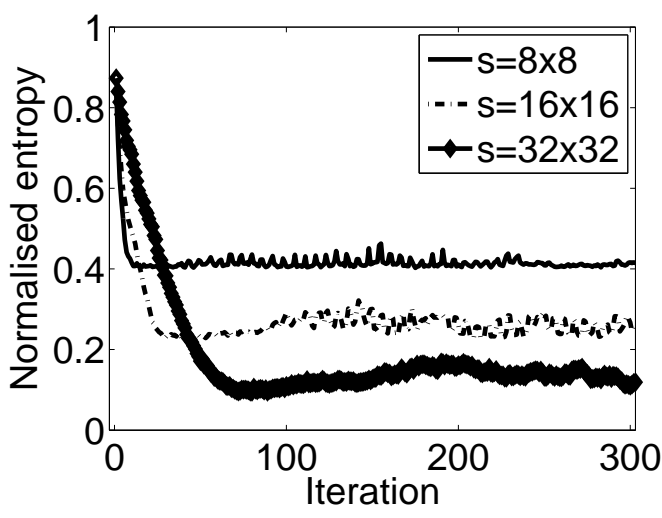

(a) Phase

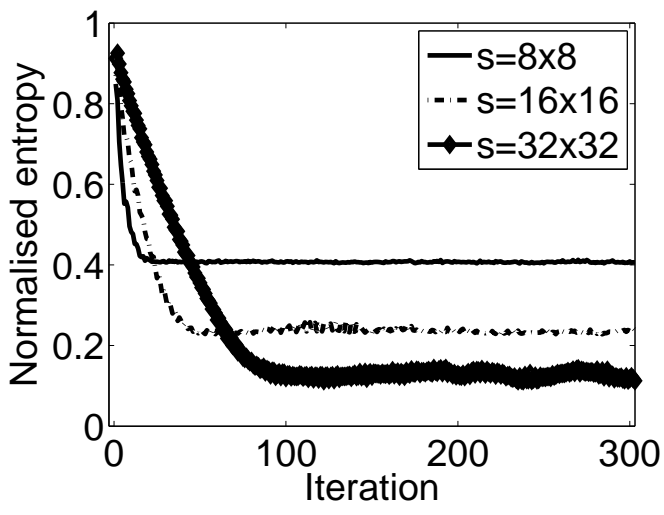

(b) Amplitude

Figure 10: Lattice size effect on the global coherence of the elements. The figures show that the smaller the lattice size, the quicker the convergence and the better the higher the normalised entropy of the phase and amplitude of the elements in the lattice.

It is also interesting to be able to understand the change of behaviour as the size of the lattice varies. From the plots in Figure 10, it can be concluded that the global phase and amplitude coherence of the lattice decrease as the size of the lattice is increased. In the cluster synchronisation case, it can be noticed that the bigger the lattice the more clusters are present (Figure 11).

\section{Conclusion}

The behaviour of a Duffing resonator coupled to a van der Pol oscillator with MEMS parameters as two coupled elements and in a square lattice have been discussed in this paper. 


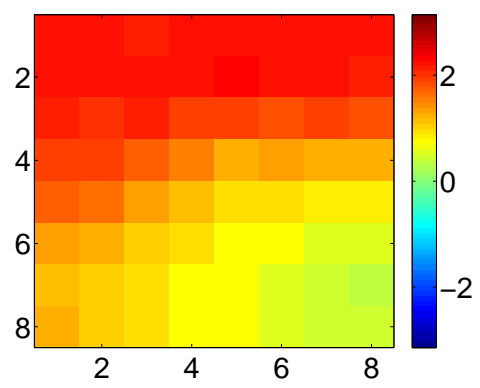

(a) $8 \times 8$

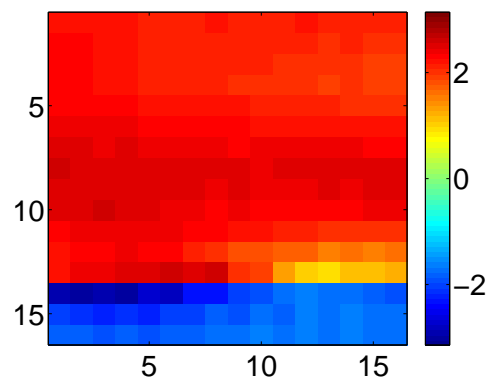

(c) $16 \times 16$

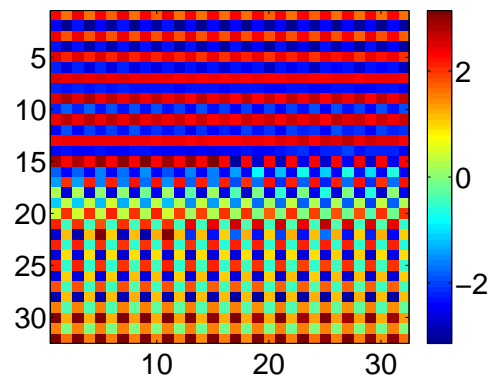

(e) $32 \times 32$

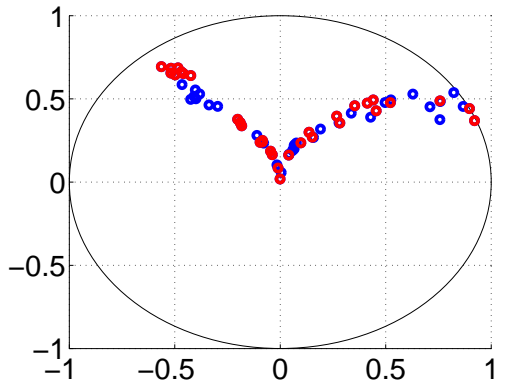

(b) $8 \times 8$

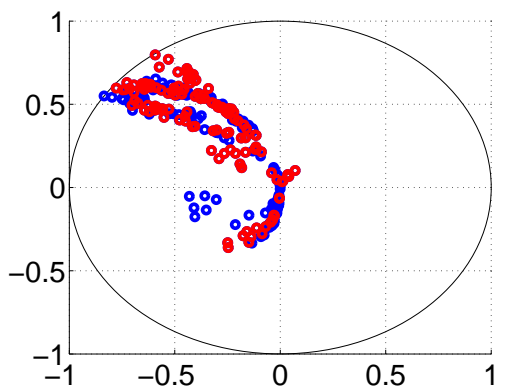

(d) $16 \times 16$

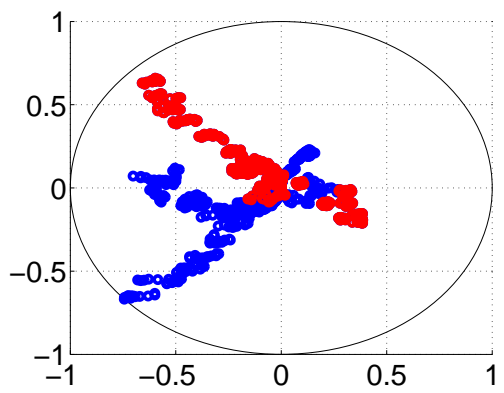

(f) $32 \times 32$

Figure 11: Instantaneous phase and polar plots of a square lattice of 32 by $32 \mathrm{x}$-coupled alternating Duffing resonators and van der Pol oscillators at a coupling strength of $\kappa=10$. The array is driven from the top edge of the lattice and the figures are plotted at $t=3600$ for different sizes of the lattice. It can be observed that for small $(8 \times 8)$ and medium (16x16) array sizes, the phases and amplitudes of the Duffing resonators and the van der Pol oscillators are in synchrony. In particular, the smaller the lattice, the better is the synchronisation. 
The two coupled elements display rich behaviours such as an hysteresis, anti-resonance, stagnant response and multiple branches in their frequency responses which have been obtained using the method of multiple time scales analysis. The stability of the system has also been investigated where two unstable branches of the Duffing resonator and van der Pol oscillator respectively have been found. Moreover the system also displays interesting transient and asymptotic behaviour. The bifurcation analysis also revealed an abrupt transition to chaos of the system.

The global and local synchronisations of a square lattice of alternating Duffing resonators and van der Pol oscillators has been studied numerically. Such a lattice can serve as a sensing device where the input signal is simulated with an external driving force that is injected to the Duffing resonators. The parameters of the resonators and oscillators that have been used come from real micro-electro-mechanical system (MEMS) devices. The effects of different system parameters have been investigated. It has been found that the higher the coupling strength value between the elements of the lattice the more synchronised they will become. In contrast, when the amplitude of the driving force is increased the elements of the lattice tend to have a random behaviour. It has been observed that with high quality factor value the synchronisation between the elements is favoured whereas a low quality factor value will result in two distinct behaviours of the resonators and oscillators.

Besides the effects of the system parameters on the behaviour of the coupled systems, a different architecture has also been investigated. More precisely, a permutation of the location of the resonators and oscillators has been carried out, resulting in a square lattice of alternating van der Pol oscillators and Duffing resonators. The behaviours of the elements in those two types of lattices are different in that the resonators and oscillators can be synchronised in the former case while they evolve independently in the latter case. The behaviour of the elements in the lattice is also dependent on its size. In particular, different clusters of synchronisation can be observed with different array sizes composed of even number of elements. However, no distinguishable clusters of sycnhronisation appear when the dimension of the lattice is odd. This suggests that the unit composed of a Duffing resonator coupled with a van der Pol oscillator represents an important entity in order to have rich 
synchronisation behaviours.

The square lattice has been driven in two different ways: from the top edge and the top left corner. It has been observed that in the first case, the resonators and oscillators tend to synchronise by rows provided that the coupling strength is high enough. In the second case, a symmetric behaviour of the elements of the lattice can be seen where the axis of symmetry is given be the diagonal joining the driven Duffing resonator on the top left corner and the last Duffing resonator on the opposite corner. Different initial conditions have also been used but the system is not sensitive to them at all.

The global and local synchronisation studies can give totally different results and one can notice that cluster synchronisation aspect provides more useful information than simply looking at the full synchronisation especially for a lattice of heterogeneous systems as in this section. Nevertheless, no cluster synchronisation characterisation is available to the best of our knowledge as opposed to the case of global synchronisation in which various synchronisation indices can be used.

\section{References}

[1] J. N. Breaa, L. M. Kayb, N. J. Kopella, Biophysical model for gamma rhythms in the olfactory bulb via subthreshold oscillations, PNAS 106 (51) (2009) 21954-21959.

[2] T. Burwick, Temporal coding: competition for coherence and new perspectives on assembly formation, in: IJCNN'09: Proceedings of the 2009 international joint conference on Neural Networks, IEEE Press, Piscataway, NJ, USA, 2009, pp. 3467-3476.

[3] M. Ursino, E. Magosso, C. Cuppini, Recognition of abstract objects via neural oscillators: interaction among topological organization, associative memory and gamma band synchronization, Trans. Neur. Netw. 20 (2) (2009) 316-335.

[4] J. A. Acebron, L. L. Bonilla, C. J. Pérez Vicente, F. Ritort, R. Spigler, The kuramoto model: A simple paradigm for synchronization phenomena, Rev. Mod. Phys. 77 (1) (2005) 137-185. 
[5] W. Wang, J.-J. E. Slotine, On partial contraction analysis for coupled nonlinear oscillators, Biological Cybernetics 92 (1) (2004) 38-53.

[6] W. Lohmiller, J.-J. E. Slotine, On contraction analysis for nonlinear systems, Automatica 34 (6) (1998) 683-696.

[7] J.-J. E. Slotine, W. Lohmiller, Modularity, evolution, and the binding problem: a view from stability theory, Neural Networks 14 (2) (2001) 137-145.

[8] C.-H. Chiu, W.-W. Lin, C.-S. Wang, Synchronization in a lattice of coupled van der Pol systems, International Journal of Bifurcation and Chaos 8 (12) (1998) 2353-2373.

[9] C.-H. Chiu, W.-W. Lin, C.-S. Wang, Synchronization in a lattice of coupled oscillators with various boundary conditions, Nonlinear Analysis 46 (2001) 213-229.

[10] V. S. Afraimovich, S. N. Chow, J. K. Hale, Synchronisation in lattices of coupled oscillators, Physica D 103 (1997) 445-1490.

[11] V. S. Afraimovich, W. W. Lin, Synchronization in lattices of coupled oscillators with Neumann/periodic boundary conditions, Dynamics and Stability Systems 13 (1998) $237-264$.

[12] J. K. Hale, Diffusive coupling, dissipative and synchronization, Journal of Dynamics and Differential Equations 9 (1) (1997) 1-52.

[13] K. Kaneko, Clustering, coding, switching, hierarchical ordering, and control in a network of chaotic elements, Physica D 41 (1990) 137-172.

[14] V. N. Belykh, E. Mosekilde, One-dimensional map lattices: synchronization, bifurcations, and chaotic structures, Physical Review E 54 (1996) 3196-3203.

[15] F. Xie, G. Hu, Spatiotemporal periodic and chaotic patterns in a two-dimensional coupled map lattice system, Physical Review E 55 (1997) 79-86.

[16] M. Hasler, Y. Maistrenko, O. Popovich, Simple example of partial synchronization of chaotic systems, Physical Review E 58 (5) (1990) 6843-6846. 
[17] V. N. Belykh, I. V. Belykh, M. Hasler, Hierarchy and stability of partially synchronous oscillations of diffusively coupled dynamical systems, Physical Review E 62 (5) (2000) $6332-6345$.

[18] V. N. Belykh, E. Mosekilde, Cluster synchronization modes in an ensemble of coupled chaotic oscillators, Physical Review E 63 (2001) 036216.

[19] M. K. Zalalutdinov, J. W. Baldwin, M. H. Marcus, R. B. Reichenbach, J. M. Parpia, B. H. Houston, Two-dimensional array of coupled nanomechanical resonators, Applied Physics Letters 88 (2006) 143504.

[20] K. Aubin, M. K. Zalalutdinov, T. Alan, R. B. Reichenbach, R. Rand, A. Z. J. M. Parpia, H. Craighead, Limit cycle oscillations in cw laser-driven nems, Journal of Microelectromechanical Systems 13 (2004) 1018-1026.

[21] L. Mendelowitz, A. Verdugo, R. Rand, Dynamics of three coupled limit cycle oscillators with application to artificial intelligence, Communications in Nonlinear Science and Numerical Simulation 14 (2009) 270-283.

[22] D. D. Bernardo, M. G. Signorini, S. Cerutti, A model of two nonlinear coupled oscillators for the study of heartbeat dynamics, International Journal of Bifurcation and Chaos 8 (10) (1998) 1975-1985.

[23] R. Rand, J. Wong, Dynamics of four coupled phase-only oscillators, Communications in Nonlinear Science and Numerical Simulation 13 (2008) 501-507.

[24] R. Rand, P. J. Holmes, Bifurcation of periodic motions in two weakly coupled van der pol oscillators, International Journal of Non-Linear Mechanics 15 (1980) 387-399.

[25] M. A. Barron, M. Sen, Synchronisation of four coupled van der pol oscillators, Nonlinear Dynamics 56 (2009) 357-367.

[26] T. Kapitaniak, Transition to hyperchaos in chaotically forced coupled oscillators, Physical Review E 47 (1993) R2975-R2978. 
[27] Y.-J. Han, Dynamics of coupled nonlinear oscillators of different attractors: van der Pol oscillator and damped Duffing oscillator, Journal of Korean Physical Society 37 (2000) 3-9.

[28] P. Woafo, J. C. Chedjou, H. B. Fotsin, Dynamics of a system consisting of a van der pol oscillator coupled to a duffing oscillator, Physical Review E 54 (6) (1996) 5929-5934.

[29] J. C. Chedjou, K. Kyamakya, I. Moussa, H.-P. Kuchenbecker, W. Mathis, Behaviour of self-sustained electromechanical transducer and routes to chaos, Journal of Vibration and Acoustics 128 (2006) 283-293.

[30] M. F. Randrianandrasana, X. Wei, D. Lowe, Collective behaviour in a square lattice of driven Duffing resonators coupled to van der Pol oscillators, in: Proceedings of the 10th IEEE International Conference on Computer and Information Technology, 2010, pp. $785-790$.

[31] A. H. Nayfeh, D. T. Mook, Nonlinear Oscillations, Wiley Classics Library, New York, 1995.

[32] X. Wei, M. F. Randrianandrasana, D. Lowe, M. Ward, Nonlinear dynamics of a periodically driven duffing resonator coupled to a van der pol oscillator, Mathematical Problems in Engineering. Accepted.

[33] P. Tass, M. G. Rosenblum, J. Weule, J. Kurths, A. S. Pikovsky, J. Volkmann, A. Schnitzler, H.-J. Freund, Detection of n:m phase locking from noisy data: application to magnetoencephalography, Physical Review Letters 81 (15) (1998) 3291-3294. 